Internat. J. Math. \& Math. Sci.

Vol. 24, No. 10 (2000) 673-689

S0161171200004671

(C) Hindawi Publishing Corp.

\title{
AN EXAMPLE OF NONSYMMETRIC SEMI-CLASSICAL FORM OF CLASS $s=1$; GENERALIZATION OF A CASE OF JACOBI SEQUENCE
}

\author{
MOHAMED JALEL ATIA
}

(Received 24 February 2000)

\begin{abstract}
We give explicitly the recurrence coefficients of a nonsymmetric semi-classical sequence of polynomials of class $s=1$. This sequence generalizes the Jacobi polynomial sequence, that is, we give a new orthogonal sequence $\left\{\hat{P}_{n}^{(\alpha, \alpha+1)}(x, \mu)\right\}$, where $\mu$ is an arbitrary parameter with $\mathfrak{R}(1-\mu)>0$ in such a way that for $\mu=0$ one has the well-known Jacobi polynomial sequence $\left\{\hat{P}_{n}^{(\alpha, \alpha+1)}(x)\right\}, n \geq 0$.
\end{abstract}

Keywords and phrases. Orthogonal polynomials, semi-classical polynomials.

2000 Mathematics Subject Classification. Primary 33C45; Secondary 42C05.

1. Introduction. Many authors [1, 2, 3] have studied semi-classical sequences of polynomials of class $s=1$. In particular, Bachène [2, page 87] gave the system fulfilled by such sequences using the structure relation and Belmehdi [3, page 272] gave the same system (in a more simple way) using directly the functional equation. This system is not linear and has not been sorted out before. The aim of this paper is to present a method that may give us some solutions.

In Section 2, we recall the general features which are needed in what follows. Section 3 is devoted to the setting of the problem, to give an integral representation and the expressions of the moments of the form $\mathscr{f}(\alpha, \alpha+1)(\mu)$ which generalizes the form $\mathscr{f}(\alpha, \alpha+1)$, where $\mathscr{g}(\alpha, \beta)$ is the Jacobi functional.

In Section 4 , the recurrence coefficients of the semi-classical sequence of polynomials orthogonal with respect to $\mathscr{f}(\alpha, \alpha+1)(\mu)$ are explicitly given using the LaguerreFreud equation of semi-classical orthogonal sequences of class $s=1$ given in [3, page 272].

2. Preliminaries. Let $\mathscr{P}$ be the vector space of polynomials with coefficients in $\mathbb{C}$ and $\mathscr{P}^{\prime}$ be its algebraic dual. We denote by $\langle u, f\rangle$ the action of $u \in \mathscr{P}^{\prime}$ on $f \in \mathscr{P}$. In particular, we denote by $(u)_{n}:=\left\langle u, x^{n}\right\rangle, n \geq 0$ the moments of $u$. Let us define the following operations on $\mathscr{P}^{\prime}$ :

- the left-multiplication of a linear functional by a polynomial

$$
\langle g u, f\rangle:=\langle u, g f\rangle, \quad f, g \in \mathscr{P}, u \in \mathscr{P}^{\prime},
$$

- the derivative of a linear functional

$$
\left\langle u^{\prime}, f\right\rangle:=-\left\langle u, f^{\prime}\right\rangle, \quad f \in \mathscr{P}, u \in \mathscr{P}^{\prime},
$$


- the homothetic of a linear functional

$$
\left\langle h_{a} u, f\right\rangle:=\left\langle u, h_{a} f\right\rangle, \quad a \in \mathbb{C}-\{0\},
$$

where

$$
\left(h_{a} f\right)(x)=f(a x), \quad f \in \mathscr{P}, u \in \mathscr{P}^{\prime},
$$

- the translation of a linear functional

$$
\left\langle\tau_{b} u, f\right\rangle:=\left\langle u, \tau_{-b} f\right\rangle, \quad b \in \mathbb{C},
$$

where

$$
\left(\tau_{b} f\right)(x)=f(x-b), \quad f \in \mathscr{P}, u \in \mathscr{P}^{\prime},
$$

- the division of a linear functional by a polynomial of first degree

$$
\left\langle(x-c)^{-1} u, f\right\rangle:=\left\langle u, \theta_{c} f\right\rangle, \quad c \in \mathbb{C},
$$

where

$$
\left(\theta_{c} f\right)(x)=\frac{f(x)-f(c)}{x-c}, \quad f \in \mathscr{P}, u \in \mathscr{P}^{\prime},
$$

- using (2.1) and (2.2) we can easily prove

$$
(f u)^{\prime}=f^{\prime} u+f u^{\prime}, \quad f \in \mathscr{P}, u \in \mathscr{P}^{\prime} .
$$

DEFINITION 2.1 (see [4]). A sequence of polynomials $\left\{\hat{P}_{n}\right\}_{n \geq 0}$ is said to be a monic orthogonal polynomial sequence with respect to the linear functional $u$ if

(i) $\operatorname{deg} \hat{P}_{n}=n$ and the leading coefficient of $\hat{P}_{n}(x)$ is equal to 1 .

(ii) $\left\langle u, \hat{P}_{n} \hat{P}_{m}\right\rangle=r_{n} \delta_{n, m}, n, m \geq 0, r_{n} \neq 0, n \geq 0$.

It is well known that a sequence of monic orthogonal polynomial satisfies a threeterm recurrence relation

$$
\begin{gathered}
\hat{P}_{0}(x)=1, \quad \hat{P}_{1}(x)=x-\beta_{0}, \\
\hat{P}_{n+2}(x)=\left(x-\beta_{n+1}\right) \hat{P}_{n+1}(x)-\gamma_{n+1} \hat{P}_{n}(x), \quad n \geq 0,
\end{gathered}
$$

with $\left(\beta_{n}, \gamma_{n+1}\right) \in \mathbb{C} \times \mathbb{C}-\{0\}, n \geq 0$.

In such conditions, we say that $u$ is regular or quasi-definite (see [4]). In what follows, we assume that the linear functionals are regular.

A shifting leaves invariant the orthogonality for the sequence $\left\{\tilde{P}_{n}\right\}_{n \geq 0}$. In fact, $\tilde{P}_{n}(x)=a^{-n} \hat{P}_{n}(a x+b), n \geq 0$, fulfills the recurrence relation [6] and [8, page 265]

$$
\begin{gathered}
\tilde{P}_{0}(x)=1, \quad \tilde{P}_{1}(x)=x-\tilde{\beta}_{0}, \\
\tilde{P}_{n+2}(x)=\left(x-\tilde{\beta}_{n+1}\right) \tilde{P}_{n+1}(x)-\tilde{\gamma}_{n+1} \tilde{P}_{n}(x), \quad n \geq 0
\end{gathered}
$$

with $\tilde{\beta}_{n}=\left(\beta_{n}-b\right) / a, \tilde{\gamma}_{n+1}=\left(\gamma_{n+1}\right) / a^{2}, n \geq 0, a \in \mathbb{C}-\{0\}$. 
Definition 2.2 (see [4]). $\left\{\hat{P}_{n}\right\}_{n \geq 0}$ (respectively, the linear functional $u$ ) is semiclassical of class $s$, if and only if the following statement holds: [6] and [7, pages 143-144].

There exist two polynomials $\psi$ of degree $p \geq 1$ and $\phi$ of degree $t \geq 0$, such that

$$
\begin{gathered}
(\phi u)^{\prime}+\psi u=0, \\
\prod_{c \in Z_{\phi}}\left(\left|\psi(c)+\phi^{\prime}(c)\right|+\left|\left\langle u, \theta_{c}(\psi)+\theta_{c}^{2}(\phi)\right\rangle\right|\right) \neq 0,
\end{gathered}
$$

where $Z_{\phi}$ is the set of zeros of $\phi$. The class of $\left\{P_{n}\right\}_{n \geq 0}$ or $u$ is given by $s=$ $\max (p-1, t-2)$ [7, pages 143-144].

If $u$ is a semi-classical functional of class $s$, then $v=\left(h_{a^{-1}} \circ \tau_{-b}\right) u$ is also semiclassical of the same class and it verifies the equation $\left(\phi_{1} v\right)^{\prime}+\psi_{1} v=0$, where

$$
\phi_{1}(x)=a^{-t} \phi(a x+b), \quad \psi_{1}(x)=a^{1-t} \psi(a x+b) .
$$

\section{Generalization of $\mathscr{f}(\alpha, \alpha+1)$ as a semi-classical sequence of class $s=1$}

3.1. Problem setting. If $u$ is a classical linear function, that is,

$$
(\phi(x) u)^{\prime}+\psi(x) u=0, \quad \operatorname{deg} \phi \leq 2, \operatorname{deg} \psi=1,
$$

from (2.9) the multiplication by $x$ gives

$$
(x \phi(x) u)^{\prime}-\phi(x) u+x \psi(x) u=0, \quad \operatorname{deg}(x \phi) \leq 3, \operatorname{deg}(x \psi-\phi) \leq 2 .
$$

If we consider the following perturbed equation

$$
\begin{gathered}
(x \phi(x) u(\mu))^{\prime}+((\mu-1) \phi(x)+x \psi(x)) u(\mu)=0, \\
\operatorname{deg}(x \phi) \leq 3, \quad \operatorname{deg}(x \psi+(\mu-1) \phi) \leq 2,
\end{gathered}
$$

we obtain, under some conditions on $\mu$, a linear functional $u(\mu)$ of class $s=1$ which generalizes the classical linear functional $u$.

\section{EXAMPLES}

(1) The Hermite case. One knows that the functional equation for the Hermite linear functional, noted $\mathscr{H}$, is [6, page 117]

$$
\mathscr{H}^{\prime}+2 x \mathscr{H}=0
$$

multiplied by $x$ gives

$$
(x \mathscr{H})^{\prime}+\left(2 x^{2}-1\right) \mathscr{H}=0 .
$$

Thus, we consider the functional equation

$$
(x \mathscr{H}(\mu))^{\prime}+\left(2 x^{2}-2 \mu-1\right) \mathscr{H}(\mu)=0
$$


which is the functional equation of the well-known generalized-Hermite linear functional, noted $\mathscr{H}(\mu)$, which is regular for $\mu \neq-n-1 / 2, n \geq 0$, and semi-classical of class $s=1$ for $\mu \neq 0$ [4] and [5, page 243]. Notice that $\mathscr{H}(0)=\mathscr{H}$.

(2) The JACobi CASE. Let us consider the functional equation for the Jacobi form, $\mathscr{F}(\alpha, \beta)$ :

$$
\left(\left(x^{2}-1\right) \mathscr{g}(\alpha, \beta)\right)^{\prime}+(-(\alpha+\beta+2) x+\alpha-\beta) \mathscr{g}(\alpha, \beta)=0
$$

multiplication by $x$ gives the following equation:

$$
\left(\left(x^{3}-x\right) \mathscr{g}(\alpha, \beta)\right)^{\prime}-\left(x^{2}-1\right) \mathscr{g}(\alpha, \beta)+\left(-(\alpha+\beta+2) x^{2}+(\alpha-\beta) x\right) \mathscr{f}(\alpha, \beta)=0 .
$$

Thus, consider

$$
\left(\left(x^{3}-x\right) \mathscr{g}(\alpha, \beta)(\mu)\right)^{\prime}+\left((\mu-\alpha-\beta-3) x^{2}+(\alpha-\beta) x+1-\mu\right) \mathscr{g}(\alpha, \beta)(\mu)=0 .
$$

Notice that $\mathscr{g}(\alpha, \beta)(0)=\mathscr{g}(\alpha, \beta)$.

(a) The Gegenbauer case $(\alpha=\beta)$. In this case (3.9) becomes

$$
\left(\left(x^{3}-x\right) \mathscr{g}(\alpha, \alpha)(\mu)\right)^{\prime}+\left((\mu-2 \alpha-3) x^{2}+1-\mu\right) \mathscr{g}(\alpha, \alpha)(\mu)=0
$$

which is the functional equation of the symmetric semi-classical functional, regular for $\mu \neq 2 n+2 \alpha+1, \mu \neq 2 n+1, n \geq 0$, of class $s=1$ for $\mu \neq 0$, and $\mathscr{f}(\alpha, \alpha)(0)=\mathscr{f}(\alpha, \alpha)$.

In fact, in [1, page 317], we have

$$
\left(\left(x^{3}-x\right) u\right)^{\prime}+2\left(-(\tilde{\alpha}+\tilde{\beta}+2) x^{2}+\tilde{\beta}+1\right) u=0
$$

and if we denote by $\left\{P_{n}\right\}_{n \geq 0}$ the sequence of monic polynomials orthogonal with respect to $u$, then $\left\{P_{n}\right\}_{n \geq 0}$ fulfills (2.10) such that

$$
\begin{gathered}
\beta_{n}=0, \\
\gamma_{2 n+1}=\frac{(n+\tilde{\beta}+1)(n+\tilde{\alpha}+\tilde{\beta}+1)}{(2 n+\tilde{\alpha}+\tilde{\beta}+1)(2 n+\tilde{\alpha}+\tilde{\beta}+2)}, \\
\gamma_{2 n+2}=\frac{(n+1)(n+\tilde{\alpha}+1)}{(2 n+\tilde{\alpha}+\tilde{\beta}+2)(2 n+\tilde{\alpha}+\tilde{\beta}+3)},
\end{gathered}
$$

for $n \geq 0$. Put

$$
-2(\tilde{\alpha}+\tilde{\beta}+2)=\mu-(2 \alpha+3), \quad 2(\tilde{\beta}+1)=1-\mu,
$$

we obtain $\left(\left(x^{3}-x\right) u\right)^{\prime}+\left((\mu-2 \alpha-3) x^{2}+1-\mu\right) u=0$ with

$$
\begin{gathered}
\beta_{n}=0, \\
\gamma_{2 n+1}=\frac{(2 n+2 \alpha+1-\mu)(2 n+1-\mu)}{(4 n+2 \alpha+1-\mu)(4 n+2 \alpha+3-\mu)}, \\
\gamma_{2 n+2}=\frac{4(n+1)(n+\alpha+1)}{(4 n+2 \alpha+3-\mu)(4 n+2 \alpha+5-\mu)},
\end{gathered}
$$

for $n \geq 0$. 
(b) $\mathscr{g}(\alpha, \alpha+1)$ case. If in (3.9), $\beta=\alpha+1$ we get

$$
\left(\left(x^{3}-x\right) \mathscr{g}(\alpha, \alpha+1)(\mu)\right)^{\prime}+\left((\mu-2 \alpha-4) x^{2}-x+1-\mu\right) \mathscr{g}(\alpha, \alpha+1)(\mu)=0 .
$$

In what follows, we will look for the regular linear functional, $\mathscr{f}(\alpha, \alpha+1)(\mu)$ which is a solution of (3.15) and we denote by $\left\{P_{n}\right\}_{n \geq 0}$ the sequence of monic orthogonal polynomials with respect to $\mathscr{f}(\alpha, \alpha+1)(\mu)$ and by $\beta_{n}, \gamma_{n}, n \geq 0$ the recurrence coefficients of $P_{n}$.

REMARK 3.1. The solutions of the functional equation (3.15) depend on the value of $(\mathscr{E}(\alpha, \alpha+1)(\mu))_{1}=\beta_{0}$, in fact,

$$
\left\langle\left(\left(x^{3}-x\right) \mathscr{g}(\alpha, \alpha+1)(\mu)\right)^{\prime}+\left((\mu-2 \alpha-4) x^{2}-x+1-\mu\right) \mathscr{g}(\alpha, \alpha+1)(\mu), 1\right\rangle=0,
$$

then, using (2.2), one has

$$
\begin{aligned}
& \left\langle\left((\mu-2 \alpha-4) x^{2}-x+1-\mu\right) \mathscr{g}(\alpha, \alpha+1)(\mu), 1\right\rangle \\
& =\left\langle\mathscr{g}(\alpha, \alpha+1)(\mu),\left((\mu-2 \alpha-4) x^{2}-x+1-\mu\right)\right\rangle \\
& =(\mu-2 \alpha-4)(\mathscr{g}(\alpha, \alpha+1)(\mu))_{2}-(\mathscr{g}(\alpha, \alpha+1)(\mu))_{1}+1-\mu=0 \text {, }
\end{aligned}
$$

but $(\mathscr{g}(\alpha, \alpha+1)(\mu))_{2}=\gamma_{1}+\beta_{0}^{2}$ and $(\mathscr{g}(\alpha, \alpha+1)(\mu))_{1}=\beta_{0}$ then

$$
(\mu-2 \alpha-4) \gamma_{1}+(\mu-2 \alpha-4) \beta_{0}^{2}-\beta_{0}+1-\mu=0 .
$$

First we search an integral representation in order to obtain $\beta_{0}$.

\subsection{An integral representation}

Proposition 3.2. An integral representation of a linear functional $\mathscr{f}(\alpha, \alpha+1)(\mu)$ is

$$
\langle\mathscr{f}(\alpha, \alpha+1)(\mu), f(x)\rangle=\frac{\Gamma((2 \alpha+3-\mu) / 2)}{\Gamma((1-\mu) / 2) \Gamma(1+\alpha)} \int_{-1}^{+1}|x|^{-\mu}\left(1-x^{2}\right)^{\alpha}(1-x) f(x) d x
$$

with $\operatorname{Re}(1-\mu)>0$, that is, $\operatorname{Re}(-u)>-1$ and $\operatorname{Re}(\alpha+1)>0$.

Proof. A solution of (3.15) has the integral representation

$$
\langle\mathscr{F}(\alpha, \alpha+1)(\mu), f\rangle=\int_{C} U(x) f(x) d x, \quad f \in \mathscr{P}
$$

if the following conditions hold [5]:

$$
\begin{gathered}
\left(\left(x^{3}-x\right) U(x)\right)^{\prime}+\left((\mu-2 \alpha-4) x^{2}-x+1-\mu\right) U(x)=0 \\
\left.\left(x^{3}-x\right) U(x) f(x)\right]_{C}=0, \quad f \in \mathscr{P}
\end{gathered}
$$

where $C$ is an acceptable integration path. We solve the first condition as a differential equation:

$$
\left(\left(x^{3}-x\right) U(x)\right)^{\prime}+\left((\mu-2 \alpha-4) x^{2}-x+1-\mu\right) U(x)=0
$$


or, equivalently,

$$
\begin{gathered}
\left(x^{3}-x\right) U^{\prime}(x)+\left((\mu-2 \alpha-1) x^{2}-x-\mu\right) U(x)=0, \\
\frac{U^{\prime}(x)}{U(x)}=-\frac{(\mu-2 \alpha-1) x^{2}-x-\mu}{x^{3}-x}=-\frac{(\mu-2 \alpha-1) x^{2}-x-\mu}{x(x-1)(x+1)} .
\end{gathered}
$$

Thus

$$
\frac{U^{\prime}(x)}{U(x)}=-\frac{\mu}{x}+\frac{(\alpha+1)}{(x-1)}+\frac{\alpha}{(x+1)}
$$

and

$$
U(x)= \begin{cases}k|x|^{-\mu}\left(1-x^{2}\right)^{\alpha}(1-x), & |x|<1, \\ 0, & |x|>1 .\end{cases}
$$

If we assume $\operatorname{Re}(1-\mu)>0, \operatorname{Re}(\alpha+1)>0$, then

$$
\left.\left.\left(x^{3}-x\right) U(x) f(x)\right]_{C}=k\left(x^{3}-x\right)|x|^{-\mu}\left(1-x^{2}\right)^{\alpha}(1-x) f(x)\right]_{-1}^{+1}=0
$$

holds.

DETERMINATION OF THE NORMALISATION FACTOR.

$$
\begin{aligned}
\langle\mathscr{g}(\alpha, \alpha+1)(\mu), 1\rangle & =k_{1} \int_{-1}^{+1}|x|^{-\mu}\left(1-x^{2}\right)^{\alpha}(1-x) d x \\
& =k_{1} \int_{-1}^{+1}|x|^{-\mu}\left(1-x^{2}\right)^{\alpha} d x \\
& =2 k_{1} \int_{0}^{+1}(x)^{-\mu}\left(1-x^{2}\right)^{\alpha} d x \\
& =2 k_{1} \frac{1}{2} B\left(\frac{1-\mu}{2}, \alpha+1\right)=1
\end{aligned}
$$

where $B(p, q)$ is the beta function. Thus, from

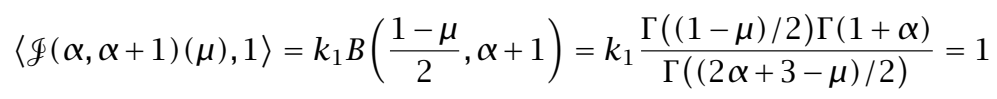

we get

$$
k_{1}=\frac{\Gamma((2 \alpha+3-\mu) / 2)}{\Gamma((1-\mu) / 2) \Gamma(1+\alpha)} .
$$

Conversely, using this integral representation, we give explicitly the expressions of the moments and the functional equation (3.15).

3.3. The expressions of the moments. Using the integral representation we have a relation between $(\mathscr{g}(\alpha, \alpha+1)(\mu))_{2 n+1}$ and $(\mathscr{g}(\alpha, \alpha+1)(\mu))_{2 n+2}$ and a relation between $(\mathscr{f}(\alpha, \alpha+1)(\mu))_{2 n+2}$ and $(\mathscr{E}(\alpha, \alpha+1)(\mu))_{2 n}$. Then, using these two relations, we obtain the functional equation.

LEMMA 3.3. Using the integral representation we have

$$
(\mathscr{F}(\alpha, \alpha+1)(\mu))_{2 n+1}=-(\mathscr{F}(\alpha, \alpha+1)(\mu))_{2 n+2}, \quad n \geq 0 .
$$




\section{Proof.}

$$
\begin{aligned}
\left\langle\mathscr{G}(\alpha, \alpha+1)(\mu), x^{2 n+1}+x^{2 n+2}\right\rangle & =k_{1} \int_{-1}^{+1}|x|^{-\mu}\left(1-x^{2}\right)^{\alpha}(1-x)\left(x^{2 n+1}+x^{2 n+2}\right) d x \\
& =k_{1} \int_{-1}^{+1} x^{2 n+1}|x|^{-\mu}\left(1-x^{2}\right)^{\alpha+1} d x=0
\end{aligned}
$$

because $x^{2 n+1}|x|^{-\mu}\left(1-x^{2}\right)^{\alpha+1}$ is an odd function.

LEMMA 3.4. Using the integral representation we have

$$
(\mathscr{g}(\alpha, \alpha+1)(\mu))_{2 n+2}=\frac{\Gamma((2 n+3-\mu) / 2) \Gamma(\alpha+1)}{\Gamma((2 n+2 \alpha+5-\mu) / 2)}
$$

and, in particular,

$$
(2 n+2 \alpha+3-\mu)(\mathscr{F}(\alpha, \alpha+1)(\mu))_{2 n+2}=(2 n+1-\mu)(\mathscr{g}(\alpha, \alpha+1)(\mu))_{2 n}, \quad n \geq 0 .
$$

Proof. From

$$
\begin{aligned}
\left\langle\mathscr{g}(\alpha, \alpha+1)(\mu), x^{2 n+2}\right\rangle & =k_{1} \int_{-1}^{+1}|x|^{-\mu}\left(1-x^{2}\right)^{\alpha}(1-x) x^{2 n+2} d x \\
& =k_{1} \int_{-1}^{+1} x^{2 n+2}|x|^{-\mu}\left(1-x^{2}\right)^{\alpha} d x
\end{aligned}
$$

taking into account that $x^{2 n+3}|x|^{-\mu}\left(1-x^{2}\right)^{\alpha}$ is an odd function,

$$
\begin{aligned}
\left\langle q(\alpha, \alpha+1)(\mu), x^{2 n+2}\right\rangle & =2 k_{1} \int_{0}^{+1} x^{2 n+2-\mu}\left(1-x^{2}\right)^{\alpha} d x \\
& =2 k_{1} \frac{1}{2} B\left(\frac{2 n+3-\mu}{2}, \alpha+1\right),
\end{aligned}
$$

where $B(p, q)$ is the beta function

$$
\begin{aligned}
\left\langle\mathscr{g}(\alpha, \alpha+1)(\mu), x^{2 n+2}\right\rangle & =\frac{\Gamma((2 n+3-\mu) / 2) \Gamma(\alpha+1)}{\Gamma((2 n+2 \alpha+5-\mu) / 2)} \\
& =\frac{2 n+1-\mu}{2 n+2 \alpha+3-\mu} \frac{\Gamma((2 n+1-\mu) / 2) \Gamma(\alpha+1)}{\Gamma((2 n+2 \alpha+3-\mu) / 2)} \\
\left\langle\mathscr{F}(\alpha, \alpha+1)(\mu), x^{2 n+2}\right\rangle & =\frac{2 n+1-\mu}{2 n+2 \alpha+3-\mu}\left\langle\mathscr{F}(\alpha, \alpha+1)(\mu), x^{2 n}\right\rangle, \quad n \geq 0 .
\end{aligned}
$$

Using (3.30) and (3.33) we can find the functional equation (3.15).

From (3.33), we have, for $n \geq 0$,

$$
(2 n+2 \alpha+3-\mu)(\mathscr{g}(\alpha, \alpha+1)(\mu))_{2 n+2}=(2 n+1-\mu)(\mathscr{g}(\alpha, \alpha+1)(\mu))_{2 n},
$$

with (3.30), one has

$$
\begin{aligned}
& (2 n+2 \alpha+4-\mu)(\mathscr{g}(\alpha, \alpha+1)(\mu))_{2 n+2} \\
& \quad=-(\mathscr{g}(\alpha, \alpha+1)(\mu))_{2 n+1}+(2 n+1-\mu)(\mathscr{g}(\alpha, \alpha+1)(\mu))_{2 n}, \quad n \geq 0 .
\end{aligned}
$$


Using (2.1) and (2.2), we get, for $n \geq 0$,

$$
\left\langle\left(\left(x^{3}-x\right) \mathscr{g}(\alpha, \alpha+1)(\mu)\right)^{\prime}+\left((\mu-2 \alpha-4) x^{2}-x-(\mu-1)\right) \mathscr{g}(\alpha, \alpha+1)(\mu), x^{2 n}\right\rangle=0 .
$$

From (3.15) and (3.33), we have, for $n \geq 0$,

$$
(2 n+2 \alpha+5-\mu)(\mathscr{g}(\alpha, \alpha+1)(\mu))_{2 n+3}=(2 n+3-\mu)(\mathscr{F}(\alpha, \alpha+1)(\mu))_{2 n+1} .
$$

Thus, taking into account (3.30), one has

$$
\begin{aligned}
& (2 n+2 \alpha+5-\mu)(\mathscr{F}(\alpha, \alpha+1)(\mu))_{2 n+3} \\
& \quad=-(\mathscr{F}(\alpha, \alpha+1)(\mu))_{2 n+2}+(2 n+2-\mu)(\mathscr{F}(\alpha, \alpha+1)(\mu))_{2 n+1}, \quad n \geq 0 .
\end{aligned}
$$

From

$$
\left\langle\left(\left(x^{3}-x\right) \mathscr{g}(\alpha, \alpha+1)(\mu)\right)^{\prime}+\left((\mu-2 \alpha-4) x^{2}-x-(\mu-1)\right) \mathscr{g}(\alpha, \alpha+1)(\mu), x^{2 n+1}\right\rangle=0, \quad n \geq 0 .
$$

equations (3.39) and (3.42) give

$$
\left\langle\left(\left(x^{3}-x\right) \mathscr{g}(\alpha, \alpha+1)(\mu)\right)^{\prime}+\left((\mu-2 \alpha-4) x^{2}-x-(\mu-1)\right) \mathscr{g}(\alpha, \alpha+1)(\mu), x^{n}\right\rangle=0, \quad n \geq 0 .
$$

Hence

$$
\left(\left(x^{3}-x\right) \mathscr{g}(\alpha, \alpha+1)(\mu)\right)^{\prime}+\left((\mu-2 \alpha-4) x^{2}-x-(\mu-1)\right) \mathscr{g}(\alpha, \alpha+1)(\mu)=0 .
$$

COROLLARY 3.5. From (3.30) and (3.33) we deduce the expressions of the moments:

$$
\begin{array}{ll}
(\mathscr{g}(\alpha, \alpha+1)(\mu))_{2 n+1}=-\prod_{i=0}^{n} \frac{(2 i+1-\mu)}{(2 \alpha+2 i+3-\mu)}, & n \geq 0, \\
(\mathscr{g}(\alpha, \alpha+1)(\mu))_{2 n+2}=-(\mathscr{L}(\alpha, \alpha+1)(\mu))_{2 n+1}, & n \geq 0 .
\end{array}
$$

4. The recurrence coefficients $\beta_{n}, \gamma_{n}, n \geq 0$

4.1. The system satisfied by recurrence coefficients of semi-classical sequences of class $s=1$. Assuming that $u$ is semi-classical of class $s=1$, then $u$ satisfies

$$
(\phi u)^{\prime}+\psi u=0
$$

with

$$
\phi(x)=\sum_{k=0}^{3} c_{k} x^{k}, \quad \sum_{k=0}^{3}\left|c_{k}\right| \neq 0, \quad \psi(x)=\sum_{k=0}^{2} a_{k} x^{k}, \quad\left|a_{2}\right|+\left|a_{1}\right| \neq 0
$$

(see [3, page 272]). Furthermore, the nonlinear system satisfied by the recurrence 
coefficients of semi-classical orthogonal sequences of class $s=1$ is

$$
\begin{gathered}
\left(a_{2}-2 n c_{3}\right)\left(\gamma_{n}+\gamma_{n+1}\right)=4 c_{3} \sum_{k=1}^{n-1} \gamma_{k}+2 \sum_{k=0}^{n-1}\left(\theta_{\beta_{n}} \phi\right)\left(\beta_{k}\right)-\psi\left(\beta_{n}\right), \quad n \geq 2, \\
\left(a_{2}-2 c_{3}\right)\left(\gamma_{1}+\gamma_{2}\right)=2\left(\theta_{\beta_{1}} \phi\right)\left(\beta_{0}\right)-\psi\left(\beta_{1}\right), \\
a_{2} \gamma_{1}=-\psi\left(\beta_{0}\right), \\
\left(a_{2}-(2 n+1) c_{3}\right) \gamma_{n+1} \beta_{n+1}=\sum_{k=0}^{n} \phi\left(\beta_{k}\right)+c_{3}\left(2 \gamma_{n}\left(n \beta_{n}+\sum_{k=0}^{n} \beta_{k}\right)+3 \sum_{k=1}^{n} \gamma_{k}\left(\beta_{k}+\beta_{k-1}\right)\right) \\
+c_{2}\left((2 n+1) \gamma_{n+1}+2 \sum_{k=1}^{n} \gamma_{k}\right)-\left(a_{2} \beta_{n}+a_{1}\right) \gamma_{n+1}, \quad n \geq 1, \\
\left(a_{2}-c_{3}\right) \gamma_{1} \beta_{1}=\phi\left(\beta_{0}\right)+\gamma_{1}\left(2 c_{3} \beta_{0}+c_{2}-a_{2} \beta_{0}-a_{1}\right) .
\end{gathered}
$$

In our case, since $c_{3}=-c_{1}=1, c_{2}=c_{0}=0$, the first equation of (4.3) becomes

$$
(\mu-2 n-2 \alpha-4)\left(\gamma_{n}+\gamma_{n+1}\right)=4 \sum_{k=1}^{n-1} \gamma_{k}+2 \sum_{k=0}^{n-1}\left(\theta_{\beta_{n}} \phi\right)\left(\beta_{k}\right)-\psi\left(\beta_{n}\right), \quad n \geq 2 .
$$

Using (2.7), we get

$$
\begin{aligned}
(\mu-2 n-2 \alpha-4) \gamma_{n+1}= & -(\mu-2 n-2 \alpha-4) \gamma_{n}+4 \sum_{k=1}^{n-1} \gamma_{k}+2 \sum_{k=0}^{n-1}\left(\beta_{n}^{2}+\beta_{k}^{2}+\beta_{n} \beta_{k}-1\right) \\
& -(\mu-2 \alpha-4) \beta_{n}^{2}+\beta_{n}-(1-\mu) \\
= & -(\mu-2 n-2 \alpha-4) \gamma_{n}+4 \sum_{k=1}^{n-1} \gamma_{k}+2 \sum_{k=0}^{n-1} \beta_{k}^{2}+2 \beta_{n} \sum_{k=0}^{n-1} \beta_{k} \\
& +(2 n+2 \alpha+4-\mu) \beta_{n}^{2}+\beta_{n}+\mu-2 n-1, \quad n \geq 2
\end{aligned}
$$

then

$$
\begin{aligned}
(\mu-2 n-2 \alpha-6) \gamma_{n+2}= & -(\mu-2 n-2 \alpha-6) \gamma_{n+1}+4 \sum_{k=1}^{n} \gamma_{k}+2 \sum_{k=0}^{n} \beta_{k}^{2}+2 \beta_{n+1} \sum_{k=0}^{n} \beta_{k} \\
& +(2 n+2 \alpha+6-\mu) \beta_{n+1}^{2}+\beta_{n+1}+\mu-2 n-3, \quad n \geq 1 .
\end{aligned}
$$

If we subtract both identities,

$$
\begin{aligned}
(\mu-2 n-2 \alpha-6) \gamma_{n+2}= & -(\mu-2 n-2 \alpha-6) \gamma_{n+1}+(\mu-2 n-2 \alpha-4) \gamma_{n+1} \\
& +(\mu-2 n-2 \alpha-4) \gamma_{n}+4 \gamma_{n}+2 \beta_{n}^{2} \\
& +2 \beta_{n+1} \sum_{k=0}^{n} \beta_{k}-2 \beta_{n} \sum_{k=0}^{n-1} \beta_{k}+(2 n+2 \alpha+6-\mu) \beta_{n+1}^{2} \\
& -(2 n+2 \alpha+4-\mu) \beta_{n}^{2}+\beta_{n+1}-\beta_{n}-2, \quad n \geq 1 .
\end{aligned}
$$


Thus the first equation of (4.3) becomes

$$
\begin{aligned}
(\mu-2 n-2 \alpha-6) \gamma_{n+2}= & 2 \gamma_{n+1}+(\mu-2 n-2 \alpha) \gamma_{n}+2 \beta_{n+1} \sum_{k=0}^{n} \beta_{k}-2 \beta_{n} \sum_{k=0}^{n-1} \beta_{k} \\
& +(2 n+2 \alpha+6-\mu) \beta_{n+1}^{2}-(2 n+2 \alpha+2-\mu) \beta_{n}^{2} \\
& +\left(\beta_{n+1}-\beta_{n}\right)-2, \quad n \geq 1 .
\end{aligned}
$$

On the other hand, (4.4) becomes

$$
\begin{aligned}
(\mu-2 n-2 \alpha-5) \gamma_{n+1} \beta_{n+1}= & \sum_{k=0}^{n} \phi\left(\beta_{k}\right)+\left(2 \gamma_{n}\left(n \beta_{n}+\sum_{k=0}^{n} \beta_{k}\right)+3 \sum_{k=1}^{n} \gamma_{k}\left(\beta_{k}+\beta_{k-1}\right)\right) \\
& +c_{2}\left((2 n+1) \gamma_{n+1}+2 \sum_{k=1}^{n} \gamma_{k}\right)-\left((\mu-2 \alpha-4) \beta_{n}-1\right) \gamma_{n+1} \\
= & \sum_{k=0}^{n}\left(\beta_{k}^{3}-\beta_{k}\right)+\left(2 \gamma_{n}\left(n \beta_{n}+\sum_{k=0}^{n} \beta_{k}\right)+3 \sum_{k=1}^{n} \gamma_{k}\left(\beta_{k}+\beta_{k-1}\right)\right) \\
& -\left((\mu-2 \alpha-4) \beta_{n}-1\right) \gamma_{n+1}, \quad n \geq 1 .
\end{aligned}
$$

Shifting the indices and subtracting, we get

$$
\begin{aligned}
(\mu-2 n-2 \alpha-7) \gamma_{n+2} \beta_{n+2}= & (\mu-2 n-2 \alpha-5) \gamma_{n+1} \beta_{n+1} \\
& +\beta_{n+1}^{3}-\beta_{n+1}+3 \gamma_{n+1}\left(\beta_{n+1}+\beta_{n}\right) \\
& +\left(2 \gamma_{n+2}\left((n+1) \beta_{n+1}+\sum_{k=0}^{n+1} \beta_{k}\right)\right) \\
& -\left(2 \gamma_{n+1}\left(n \beta_{n}+\sum_{k=0}^{n} \beta_{k}\right)\right)-\left((\mu-2 \alpha-4) \beta_{n+1}-1\right) \gamma_{n+2} \\
& +\left((\mu-2 \alpha-4) \beta_{n}-1\right) \gamma_{n+1}, \quad n \geq 0 .
\end{aligned}
$$

Thus, from (4.9) and (4.11) we have the following.

\section{PROPOSITION 4.1.}

$$
\begin{aligned}
& (\mu-2 n-2 \alpha-6) \gamma_{n+2} \\
& =2 \gamma_{n+1}+(\mu-2 n-2 \alpha) \gamma_{n}+2 \beta_{n+1} \sum_{k=0}^{n} \beta_{k}-2 \beta_{n} \sum_{k=0}^{n-1} \beta_{k} \\
& \quad+(2 n+2 \alpha+6-\mu) \beta_{n+1}^{2}-(2 n+2 \alpha+2-\mu) \beta_{n}^{2}+\left(\beta_{n+1}-\beta_{n}\right)-2, \quad n \geq 1 \\
& (\mu-2 \alpha-6)\left(\gamma_{1}+\gamma_{2}\right)=2\left(\beta_{1}^{2}+\beta_{0} \beta_{1}+\beta_{0}^{2}-1\right)-(\mu-2 \alpha-4) \beta_{1}^{2}+\beta_{1}-(1-\mu) \\
& (\mu-2 \alpha-4) \gamma_{1}=-(\mu-2 \alpha-4) \beta_{0}^{2}+\beta_{0}-(1-\mu) . \\
& (\mu-2 n-2 \alpha-7) \gamma_{n+2} \beta_{n+2} \\
& \quad=\beta_{n+1}^{3}-\beta_{n+1}+(2 n+2 \alpha+8-\mu) \gamma_{n+2} \beta_{n+1}+(\mu-2 n-2 \alpha-2) \gamma_{n+1} \beta_{n+1} \\
& \quad+(\mu-2 n-2 \alpha-1) \gamma_{n+1} \beta_{n}+\left(2 \sum_{k=0}^{n} \beta_{k}+1\right)\left(\gamma_{n+2}-\gamma_{n+1}\right), \quad n \geq 0 \\
& (\mu-2 \alpha-5) \gamma_{1} \beta_{1}=\beta_{0}^{3}-\beta_{0}+\gamma_{1}\left(2 \beta_{0}-(\mu-2 \alpha-4) \beta_{0}+1\right) .
\end{aligned}
$$


Next, we will find the expressions of the recurrence parameters $\beta_{n}, \gamma_{n}, n \geq 0$.

Since $\beta_{0}=-(\mu-1) /(\mu-2 \alpha-3)$ and from (4.14) we have

$$
\begin{aligned}
\gamma_{1} & =-\frac{(\mu-2 \alpha-4) \beta_{0}^{2}+\beta_{0}-(1-\mu)}{\mu-2 \alpha-4} \\
& =-\beta_{0}^{2}+\frac{\beta_{0}}{\mu-2 \alpha-4}+\frac{\mu-1}{\mu-2 \alpha-4} \\
& =-\left(\frac{\mu-1}{\mu-2 \alpha-3}\right)^{2}-\frac{\mu-1}{(\mu-2 \alpha-3)(\mu-2 \alpha-4)}+\frac{\mu-1}{\mu-2 \alpha-4} \\
& =-\left(\frac{\mu-1}{\mu-2 \alpha-3}\right)^{2}+\frac{\mu-1}{\mu-2 \alpha-3}=2 \frac{(\alpha+1)(1-\mu)}{(2 \alpha+3-\mu)^{2}} .
\end{aligned}
$$

Using (4.16), (4.17) gives

$$
\beta_{1}=\frac{\beta_{0}^{3}-\beta_{0}+\gamma_{1}\left(-(\mu-2 \alpha-6) \beta_{0}+1\right)}{(\mu-2 \alpha-5) \gamma_{1}}=\frac{\mu(\mu-2 \alpha-4)-(2 \alpha+1)}{(2 \alpha+3-\mu)(2 \alpha+5-\mu)}
$$

With $\beta_{0}, \beta_{1}$, and $\gamma_{1},(4.13)$ gives

$$
\gamma_{2}=-\gamma_{1}+\frac{2\left(\beta_{1}^{2}+\beta_{0} \beta_{1}+\beta_{0}^{2}-1\right)-(\mu-2 \alpha-4) \beta_{1}^{2}+\beta_{1}-(1-\mu)}{\mu-2 \alpha-6}=\frac{2(2 \alpha+3-\mu)}{(2 \alpha+5-\mu)^{2}} .
$$

With $\beta_{0}, \beta_{1}, \gamma_{1}$, and $\gamma_{2},(4.15)$ and some easy computations

$$
\beta_{2}=-\frac{\mu(\mu-2 \alpha-6)+(2 \alpha+1)}{(2 \alpha+5-\mu)(2 \alpha+7-\mu)}
$$

Proposition 4.2. Assuming

$$
\begin{aligned}
& \beta_{0}=-\frac{\mu-1}{\mu-2 \alpha-3}, \\
& \beta_{n+1}=(-1)^{n} \frac{\mu(\mu-2 n-2 \alpha-4)+(-1)^{n+1}(2 \alpha+1)}{(2 n+2 \alpha+3-\mu)(2 n+2 \alpha+5-\mu)}, \\
& \gamma_{2 n+1}=2 \frac{(n+\alpha+1)(2 n+1-\mu)}{(4 n+2 \alpha+3-\mu)^{2}}, \\
& \gamma_{2 n+2}=\frac{(2 n+2)(2 n+2 \alpha+3-\mu)}{(4 n+2 \alpha+5-\mu)^{2}},
\end{aligned}
$$

for $n \geq 0$ and assume $\mu \neq 2 n+1, \mu \neq 2 n+2 \alpha+1, \alpha \neq-n-1, n \geq 0$.

LEMMA 4.3. If $E_{n}=\sum_{k=0}^{n} \beta_{k}, n \geq 0$, then

$$
E_{2 n}=-\left(\frac{2 n+1-\mu}{4 n+2 \alpha+3-\mu}\right), \quad E_{2 n+1}=-\frac{2 n+2}{4 n+2 \alpha+5-\mu}, \quad n \geq 0 .
$$


Proof. $E_{0}=\beta_{0}$. For $n \geq 0$, we have

$$
\begin{aligned}
& E_{2 n+1}= \sum_{k=0}^{n}\left(\beta_{2 k}+\beta_{2 k+1}\right) \\
&= \sum_{k=0}^{n}-\frac{\mu(\mu-4 k-2 \alpha-2)+2 \alpha+1}{(4 k+2 \alpha+1-\mu)(4 k+2 \alpha+3-\mu)} \\
& \quad+\frac{\mu(\mu-4 k-2 \alpha-4)-2 \alpha-1}{(4 k+2 \alpha+3-\mu)(4 k+2 \alpha+5-\mu)} \\
&= \sum_{k=0}^{n}-\frac{1}{2} \frac{\mu-2 \alpha-1}{(-4 k-2 \alpha-1+\mu)}-\frac{1}{2} \frac{\mu+2 \alpha+1}{(-4 k-2 \alpha-3+\mu)} \\
& \quad+\frac{1}{2} \frac{\mu+2 \alpha+1}{(-4 k-2 \alpha-3+\mu)}+\frac{1}{2} \frac{\mu-2 \alpha-1}{(-4 k-2 \alpha-5+\mu)} \\
&= \sum_{k=0}^{n}-\frac{1}{2} \frac{\mu-2 \alpha-1}{(-4 k-2 \alpha-1+\mu)}+\frac{1}{2} \frac{\mu-2 \alpha-1}{(-4 k-2 \alpha-5+\mu)} \\
&=-\frac{1}{2} \frac{\mu-2 \alpha-1}{(-2 \alpha-1+\mu)}+\frac{1}{2} \frac{\mu-2 \alpha-1}{(-4 n-2 \alpha-5+\mu)} \\
&=-\frac{\mu-2 \alpha-1}{2}\left(\frac{1}{-2 \alpha-1+\mu}-\frac{1}{-4 n-2 \alpha-5+\mu}\right) \\
&=-\frac{(\mu-2 \alpha-1)(-4 n-2 \alpha-5+\mu+2 \alpha+1-\mu)}{2(-2 \alpha-1+\mu)(-4 n-2 \alpha-5+\mu)} \\
&=-\frac{\mu-2 \alpha-1}{2}\left(\frac{-4 n-4}{(-2 \alpha-1+\mu)(-4 n-2 \alpha-5+\mu)}\right) \\
& E_{2 n+1}=-\frac{2 n+2}{(4 n+2 \alpha+5-\mu)}, \quad \mu \neq 4 n+2 \alpha+5, n \geq 0 . \\
&
\end{aligned}
$$

Calculus of

$$
\begin{aligned}
E_{2 n+2}= & E_{2 n+1}+\beta_{2 n+2} \\
= & -\frac{2 n+2}{4 n+2 \alpha+5-\mu}-\frac{\mu(\mu-4 n-2 \alpha-6)+2 \alpha+1}{(4 n+2 \alpha+5-\mu)(4 n+2 \alpha+7-\mu)} \\
= & -\frac{1}{4 n+2 \alpha+5-\mu}\left(2 n+2+\frac{\mu(\mu-2 \alpha-4 n-6)+2 \alpha+1}{4 n+2 \alpha+7-\mu}\right) \\
= & -\frac{1}{4 n+2 \alpha+5-\mu} \\
& \times\left(\frac{(2 n+2)(4 n+2 \alpha+7)-(2 n+2) \mu+\mu(\mu-2 \alpha-4 n-6)+2 \alpha+1}{4 n+2 \alpha+7-\mu}\right) \\
= & -\frac{1}{4 n+2 \alpha+5-\mu}\left(\frac{\mu^{2}-(6 n+2 \alpha+8) \mu+(2 n+2)(4 n+2 \alpha+7)+2 \alpha+1}{4 n+2 \alpha+7-\mu}\right) \\
= & -\frac{1}{4 n+2 \alpha+5-\mu}\left(\frac{(4 n+2 \alpha+5-\mu)(2 n+3-\mu)}{4 n+2 \alpha+7-\mu}\right), \\
E_{2 n+2}= & -\frac{2 n+3-\mu}{4 n+2 \alpha+7-\mu}, \quad \mu \neq 4 n+2 \alpha+7, n \geq 0 .
\end{aligned}
$$


Proof of Proposition 4.2. Suppose that we have

$$
\begin{aligned}
& \beta_{0}=-\frac{\mu-1}{\mu-2 \alpha-3}, \\
& \beta_{2 k+1}=\frac{\mu(\mu-4 k-2 \alpha-4)-(2 \alpha+1)}{(4 k+2 \alpha+3-\mu)(4 k+2 \alpha+5-\mu)}, \quad 0 \leq k \leq n, \\
& \beta_{2 k}=-\frac{\mu(\mu-4 k-2 \alpha-2)+(2 \alpha+1)}{(4 k+2 \alpha+1-\mu)(4 k+2 \alpha+3-\mu)}, \quad 1 \leq k \leq n, \\
& \gamma_{2 k+1}=2 \frac{(k+\alpha+1)(2 k+1-\mu)}{(4 k+2 \alpha+3-\mu)^{2}}, \quad 0 \leq k \leq n, \\
& \gamma_{2 k+2}=\frac{(2 k+2)(2 k+2 \alpha+3-\mu)}{(4 k+2 \alpha+5-\mu)^{2}}, \quad 0 \leq k \leq n-1,
\end{aligned}
$$

and, using (4.10), (4.13), we prove by induction $\beta_{2 n+2}, \beta_{2 n+3}, \gamma_{2 n+2}$, and $\gamma_{2 n+3}$. The substitution $n \rightarrow 2 n$ in (4.10) gives

$$
\begin{aligned}
(\mu-2 \alpha-4 n-6) \gamma_{2 n+2}= & 2 \gamma_{2 n+1}+(\mu-2 \alpha-4 n) \gamma_{2 n}+2 \beta_{2 n+1} E_{2 n}-2 \beta_{2 n} E_{2 n-1} \\
& +(4 n-\mu+2 \alpha+6) \beta_{2 n+1}^{2}-(4 n-\mu+2 \alpha+2) \beta_{2 n}^{2} \\
& +\left(\beta_{2 n+1}-\beta_{2 n}\right)-2, \quad n \geq 1 .
\end{aligned}
$$

We suppose known $\gamma_{2 n+1}, \gamma_{2 n}, \beta_{2 n+1}, \beta_{2 n}, E_{2 n}$, and $E_{2 n-1}$ and then we evaluate $\gamma_{2 n+2}$ for the proof by recurrence; because of cumbersome computation, using Maple. The substitution $n \rightarrow 2 n+1$ in (4.10) gives (see appendix)

$$
\begin{aligned}
(\mu-2 \alpha-4 n-8) \gamma_{2 n+3}= & 2 \gamma_{2 n+2}+(\mu-2 \alpha-4 n-2) \gamma_{2 n+1}+2 \beta_{2 n+2} E_{2 n+1}-2 \beta_{2 n+1} E_{2 n} \\
& +(4 n-\mu+2 \alpha+8) \beta_{2 n+2}^{2}-(4 n-\mu+2 \alpha+4) \beta_{2 n+1}^{2} \\
& +\left(\beta_{2 n+2}-\beta_{2 n+1}\right)-2, \quad n \geq 0 .
\end{aligned}
$$

The substitution $n \rightarrow 2 n+1$ in (4.13) gives (see appendix)

$$
\begin{aligned}
(\mu-2 \alpha-4 n-7) \gamma_{2 n+2} \beta_{2 n+2}= & \beta_{2 n+1}^{3}-\beta_{2 n+1}+(-\mu+2 \alpha+4 n+5) \beta_{2 n+1} \gamma_{2 n+2} \\
& -(-\mu+2 \alpha+4 n+2) \beta_{2 n+1} \gamma_{2 n+1} \\
& -(-\mu+2 \alpha+4 n+1) \beta_{2 n} \gamma_{2 n+1} \\
& +\left(2 E_{2 n}+1\right)\left(\gamma_{2 n+2}-\gamma_{2 n+1}\right), \quad n \geq 0 .
\end{aligned}
$$

Finally, the substitution $n \rightarrow 2 n+2$ in (4.13) gives (see appendix)

$$
\begin{aligned}
(\mu-2 \alpha-4 n-5) \gamma_{2 n+3} \beta_{2 n+3}= & \beta_{2 n+2}^{3}-\beta_{2 n+2}+(-\mu+2 \alpha+4 n+10) \beta_{2 n+2} \gamma_{2 n+3} \\
& -(-\mu+2 \alpha+4 n+4) \beta_{2 n+2} \gamma_{2 n+2} \\
& -(-\mu+2 \alpha+4 n+3) \beta_{2 n+1} \gamma_{2 n+2} \\
& +\left(2 E_{2 n+1}+1\right)\left(\gamma_{2 n+3}-\gamma_{2 n+2}\right), \quad n \geq 0 .
\end{aligned}
$$


REMARKS. (1) An homothetie of rapport -1 gives a generalization of $\mathscr{f}(\alpha+1, \alpha)$, with (2.11), (2.13) we have

$$
\begin{aligned}
& \left(\left(x^{3}-x\right) u\right)^{\prime}+\left((\mu-2 \alpha-4) x^{2}+x-(\mu-1)\right) u=0, \\
& \beta_{0}=\frac{\mu-1}{\mu-2 \alpha-3}, \\
& \beta_{n+1}=(-1)^{n+1} \frac{\mu(\mu-2 n-2 \alpha-4)+(-1)^{n+1}(2 \alpha+1)}{(2 n+2 \alpha+3-\mu)(2 n+2 \alpha+5-\mu)}, \\
& \gamma_{2 n+1}=2 \frac{(n+\alpha+1)(2 n+1-\mu)}{(4 n+2 \alpha+3-\mu)^{2}}, \\
& \gamma_{2 n+2}=\frac{(2 n+2)(2 n+2 \alpha+3-\mu)}{(4 n+2 \alpha+5-\mu)^{2}},
\end{aligned}
$$

for $n \geq 0$.

(2) For $\mu=2 \alpha+4$, we have an apparent particular case

$$
\begin{aligned}
& \left(\left(x^{3}-x\right) u\right)^{\prime}+(x-(2 \alpha+3)) u=0, \\
& \beta_{0}=-(2 \alpha+3), \\
& \beta_{n+1}=(-1)^{n} \frac{(2 \alpha+4)(-2 n)+(-1)^{n+1}(2 \alpha+1)}{(2 n-1)(2 n+1)}, \\
& \gamma_{2 n+1}=2 \frac{(n+\alpha+1)(2 n-2 \alpha-3)}{(4 n-1)^{2}}, \\
& \gamma_{2 n+2}=\frac{(2 n+2)(2 n-1)}{(4 n+1)^{2}},
\end{aligned}
$$

for $n \geq 0$.

5. Appendix. In this appendix, we give both the input and output of the Maple programme used to carry out the computations of Section 4 .

$>$ restart;

$>$ beta $0:=-(m u-1) /(m u-2 * a 1 p h a-3)$;

$$
\beta 0:=-\frac{\mu-1}{\mu-2 \alpha-3}
$$

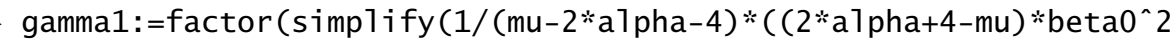
+beta0+mu-1)));

$$
\gamma 1:=-2 \frac{(1+\alpha)(\mu-1)}{(\mu-2 \alpha-3)^{2}}
$$

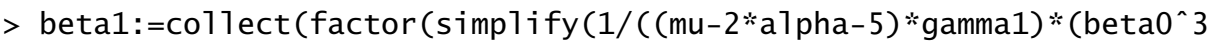
-beta $0+$ gamma1*(-(mu-2*a1pha-6)*beta $0+1))))$, mu $)$;

$\mathrm{E} 1:=\operatorname{col} 1$ ect (simplify (beta0+beta1), mu);

$$
\begin{gathered}
\beta 1:=\frac{\mu^{2}+(-2 \alpha-4) \mu-2 \alpha-1}{(\mu-2 \alpha-3)(\mu-2 \alpha-5)} \\
E 1:=\frac{2}{\mu-2 \alpha-5}
\end{gathered}
$$


$>$ gamma $2:=$ factor $($ simp 1 ify $(-$ gamma $1+1 /($ mu $-2 * a 1$ pha -6$) *(2 *$ beta $1 ` 2$

$+2 *$ beta $0 *$ beta $1+2 *$ beta $0 \wedge 2-2-(\mathrm{mu}-2 * \mathrm{a} 1$ pha -4$) *$ beta $1 \wedge 2+$ beta $1-1+\mathrm{mu}))$ );

$$
\gamma 2:=-2 \frac{\mu-2 \alpha-3}{(\mu-2 \alpha-5)^{2}}
$$

$>$ beta $2:=\operatorname{col} 1$ ect (factor (simp1ify $(1 /((\mathrm{mu}-2 * a 1$ pha $-4-3) *$ gamma 2$)$

*(beta1^3-beta1+(4-mu+2*a1pha+4)*beta1*gamma $2+(2+m u-2 * a 1$ pha -4$)$

*beta1*gamma $1+(3+$ mu $-2 *$ a 1 pha -4$) *$ beta $0 *$ gamma $1+(2 *$ beta $0+1)$

*(gamma2-gamma1)))) , mu) ; E2:=col1ect (simp1 i fy (beta2+E1), mu);

$$
\begin{gathered}
\beta 2:=-\frac{\mu^{2}+(-2 \alpha-6) \mu+2 \alpha+1}{(\mu-2 \alpha-5)(\mu-2 \alpha-7)} \\
E 2:=-\frac{\mu-3}{\mu-2 \alpha-7}
\end{gathered}
$$

$>$ gamma $:=$ factor $($ simplify $(1 /($ mu $-2 * a 1$ pha -8$) *(2 *$ gamma $2+(m u-2 * a 1$ pha -2$)$ *gamma1+2*beta2*E1-2*beta1*beta $0+(8-\mathrm{mu}+2 * a 1$ pha $) *$ beta2 2

- (2*a1pha+4-mu)*beta1^2+beta2-beta1-2)));

$$
\gamma 3:=-2 \frac{(\alpha+2)(\mu-3)}{(\mu-2 \alpha-7)^{2}}
$$

$>$ beta3:=col1ect (factor (simplify $(1 /(($ mu $-2 * a 1 p h a-4-5) *$ gamma3)

*(beta2^3-beta2+(6-mu+2*a1pha+4)*beta $2 *$ gamma3+(mu-2*a1pha-4)

*beta $2 *$ gamma $2+(1+m u-2 * a 1$ pha -4$) *$ beta $1 *$ gamma $2+(2 * E 1+1)$

*(gamma3-gamma2)))), mu) ; E3:=col1ect (simp1 ify (beta3+E2), mu) ;

$$
\begin{gathered}
\beta 3:=\frac{\mu^{2}+(-2 \alpha-8) \mu-2 \alpha-1}{(\mu-2 \alpha-7)(\mu-2 \alpha-9)} \\
E 3:=\frac{4}{\mu-2 \alpha-9}
\end{gathered}
$$

$>$ gamma $4:=$ factor $($ simp 1 ify $(1 /($ mu $-2 * a 1$ pha -10$) *(2 *$ gamma $3+(m u-2 * a 1$ pha -4$)$ *gamma $2+2 *$ beta3*E2-2*beta $2 * E 1+(10-m u+2 * a 1$ pha $) *$ beta3 2

- $(2 * a 1$ pha+6-mu $) *$ beta2 ${ }^{\wedge} 2+$ beta3-beta $\left.\left.\left.2-2\right)\right)\right)$;

$$
\gamma 4:=-4 \frac{\mu-2 \alpha-5}{(\mu-2 \alpha-9)^{2}}
$$

$>$ beta $4:=\operatorname{col} 1$ ect (factor (simplify $(1 /($ (mu- $2 * a 1$ pha $-4-4 * 1-3) *$ gamma 4$)$ *(beta3^3-beta3+(4*1+4-mu+2*a1 pha+4)*beta $3 *$ gamma 4 $+(-4 * 1+2+$ mu $-2 *$ a 1 pha -4$) *$ beta $3 *$ gamma $3+(-4 * 1+3+$ mu $-2 *$ a 1 pha -4$)$

*beta $2 *$ gamma $3+(2 * \mathrm{E} 2+1) *($ gamma $4-$ gamma 3$)))), \mathrm{mu})$;

$$
\beta 4:=-\frac{\mu^{2}+(-10-2 \alpha) \mu+2 \alpha+1}{(\mu-2 \alpha-9)(\mu-2 \alpha-11)}
$$

$>$ gamma $2 n:=2 * n *(2 * n+2 * a 1$ pha $+1-m u) /(4 * n+2 * a 1$ pha $+1-m u) \wedge 2$;

$$
\gamma 2 n:=2 \frac{n(2 n+2 \alpha+1-\mu)}{(4 n+2 \alpha+1-\mu)^{2}}
$$


> gamma2np1: $=2 *(n+a 1 p h a+1) *(2 * n+1-m u) /(4 * n+2 * a 1 p h a+3-m u) \wedge 2$;

$$
\gamma 2 n p 1:=2 \frac{(n+\alpha+1)(2 n+1-\mu)}{(4 n+2 \alpha+3-\mu)^{2}}
$$

$>$ beta $2 \mathrm{n}:=-(m u *(m u-4 * n-2 * a 1 p h a-2)+2 * a 1$ pha +1$) /((4 * n+2 * a 1 p h a+1-m u)$

$*(4 * n+2 * a 1 p h a+3-m u))$;

$$
\beta 2 n:=-\frac{\mu(\mu-4 n-2 \alpha-2)+2 \alpha+1}{(4 n+2 \alpha+1-\mu)(4 n+2 \alpha+3-\mu)}
$$

$>$ convert(beta2n, parfrac, $n)$;

$$
-1 / 2 \frac{-2 \alpha-1+\mu}{-4 n-2 \alpha-1+\mu}-1 / 2 \frac{2 \alpha+1+\mu}{-4 n-2 \alpha-3+\mu}
$$

$>$ beta $2 n p 1:=(m u *(m u-4 * n-2 * a 1 p h a-4)-2 * a 1 p h a-1) /((4 * n+2 * a 1 p h a+3-m u)$ $*(4 * n+2 * a 1 p h a+5-m u))$;

$$
\beta 2 n p 1:=\frac{\mu(\mu-4 n-2 \alpha-4)-2 \alpha-1}{(4 n+2 \alpha+3-\mu)(4 n+2 \alpha+5-\mu)}
$$

$>$ convert(beta2np1, parfrac, $\mathrm{n})$;

$$
1 / 2 \frac{2 \alpha+1+\mu}{-4 n-2 \alpha-3+\mu}+1 / 2 \frac{-2 \alpha-1+\mu}{-4 n-2 \alpha-5+\mu}
$$

$>E 2 n:=-(2 * n+1-m u) /(4 * n+2 * a 1 p h a+3-m u) ;$

$$
E 2 n:=-\frac{2 n+1-\mu}{4 n+2 \alpha+3-\mu}
$$

$>$ E2np1: $=-(2 * n+2) /(4 * n+2 * a 1 p h a+5-m u) ;$

E2nm1: $=-(2 * n) /(4 * n+2 * a 1 p h a+1-m u)$;

$$
\begin{aligned}
& E 2 n p 1:=-\frac{2 n+2}{4 n+2 \alpha+5-\mu} \\
& E 2 n m 1:=-2 \frac{n}{4 n+2 \alpha+1-\mu}
\end{aligned}
$$

$>$ gamma2np2:=factor (simpli fy $(1 /($ mu- $2 * a 1 p h a-4 * n-6)$

* 2 *gamma $2 n p 1+($ mu $-2 *$ a 1 pha $-4 * n) *$ gamma $2 n+2 *$ beta $2 n p 1 * E 2 n-2$

*beta $2 n * E 2 n m 1+(4 * n+6-m u+2 * a 1 p h a) *$ beta $2 n p 1 * 2$

$-(4 * n+2 * a 1$ pha $+2-m u) *$ beta2n^2+beta2np1-beta2n-2)));

$$
\gamma 2 n p 2:=-2 \frac{(n+1)(-2 n+\mu-3-2 \alpha)}{(-4 n-2 \alpha-5+\mu)^{2}}
$$

$>$ beta2np2:=factor (simplify $(1 /((\mathrm{mu}-2 * a 1$ pha- $4-4 * n-3) *$ gamma2np 2$)$ *(beta2np1^3-beta2np1+(4*n+4-mu+2*a1pha+4)*beta2np1*gamma2np2 $+(-4 * n+2+$ mu $-2 * a 1$ pha -4$) *$ beta $2 n p 1 *$ gamma $2 n p 1+(-4 * n+3+$ mu $-2 * a 1$ pha -4$)$ *beta2n*gamma2np1+(2*E2n+1)*(gamma2np2-gamma2np1))));

$$
\beta 2 n p 2:=-\frac{\mu^{2}-2 \mu \alpha-4 \mu n-6 \mu+1+2 \alpha}{(-4 n-2 \alpha-5+\mu)(\mu-2 \alpha-7-4 n)}
$$


$>$ gamma2np3:=factor (simplify $(1 /($ mu $-2 * a 1 p h a-4 * n-8)$

*(2*gamma2np2+(mu-2*a1pha $-4 * n-2) *$ gamma2np1+2*beta2np2*E2np1-2

*beta2np1*E2n+(4*n+8-mu+2*a1pha)*beta2np2^2-(4*n+2*a1pha+4-mu)

*beta2np1^2+beta2np2-beta2np1-2)));

$$
\gamma 2 n p 3:=-2 \frac{(n+2+\alpha)(\mu-2 n-3)}{(\mu-2 \alpha-7-4 n)^{2}}
$$

$>$ beta2np3:=(factor (simp1ify $(1 /($ (mu- $2 * a 1 p h a-4-4 * n-5) *$ gamma2np 3$)$

*(beta2np2^3-beta2np2+(4*n+6-mu+2*a1pha+4)*beta2np2*gamma2np3

$+(-4 * n+m u-2 * a 1$ pha -4$) *$ beta2np $2 *$ gamma2np $2+(-4 * n+1+m u-2 * a 1$ pha -4$)$

*beta2np1*gamma2np2+(2*E2np1+1)*(gamma2np3-gamma2np2)))) ;

$$
\beta 2 n p 3:=\frac{\mu-4 \mu n-8 \mu-2 \mu \alpha-2 \alpha-1}{(\mu-2 \alpha-7-4 n)(\mu-2 \alpha-9-4 n)}
$$

\section{REFERENCES}

[1] J. Alaya and P. Maroni, Symmetric Laguerre-Hahn forms of class $s=1$, Integral Transform. Spec. Funct. 4 (1996), no. 4, 301-320. MR 98m:42032. Zbl 865.42021.

[2] M. Bachene, Les polynômes orthogonaux semi-classiques de classe zéro et de classe un, Tech. report, Universite de Paris (Pierre et Marie Curie), Paris, France, 1985, Thèse de troisième cycle.

[3] S. Belmehdi, On semi-classical linear functionals of class $s=1$. Classification and integral representations, Indag. Math. (N.S.) 3 (1992), no. 3, 253-275. MR 94e:33038. Zbl 783.33003.

[4] T. S. Chihara, An introduction to orthogonal polynomials, Mathematics and its Applications, vol. 13, Gordon and Breach Science Publishers, New York, London, Paris, 1978. MR 58\#1979. Zbl 389.33008.

[5] P. Maroni, Sur la suite de polynômes orthogonaux associée à la forme $u=\delta_{c}+\lambda(x-c)^{-1} L$ [On the sequence of orthogonal polynomials associated with the form $u=\delta_{c}+\lambda(x-$ $\left.c)^{-1} L\right]$, Period. Math. Hungar. 21 (1990), no. 3, 223-248 (French). MR 92c:42025. Zbl 732.42015.

[6] _ Une théorie algébrique des polynômes orthogonaux. Application aux polynômes orthogonaux semi-classiques [An algebraic theory of orthogonal polynomials. Application to semiclassical orthogonal polynomials], Orthogonal Polynomials and their Applications (Erice, 1990) (Basel), IMACS Ann. Comput. Appl. Math., vol. 9, Baltzer, 1991, pp. 95-130 (French). MR 95i:42018. Zbl 950.49938.

[7] _ Variations around classical orthogonal polynomials. Connected problems, J. Comput. Appl. Math. 48 (1993), no. 1-2, 133-155, Proceedings of the Seventh Spanish Symposium on Orthogonal Polynomials and Applications (VII SPOA) (Granada, 1991). MR 94k:33013. Zbl 790.33006.

[8] _ Modified classical orthogonal polynomials associated with oscillating functionsopen problems, Appl. Numer. Math. 15 (1994), no. 2, 259-283, Innovative methods in numerical analysis (Bressanone, 1992). MR 96d:42037. Zbl 826.42020.

Mohamed Jalel Atia: Faculté des Sciences de Gabès, 6029 Route de Mednine Gabès, TUNISIA

E-mail address: jale1.atia@fsg.rnu.tn 


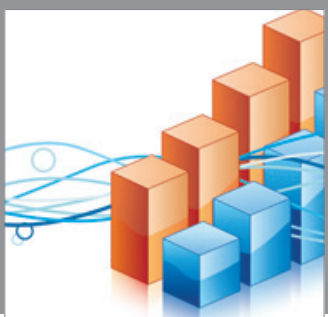

Advances in

Operations Research

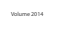

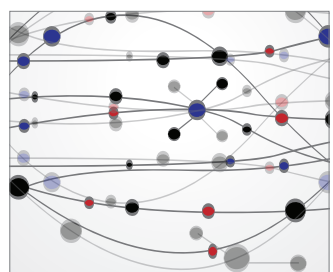

\section{The Scientific} World Journal
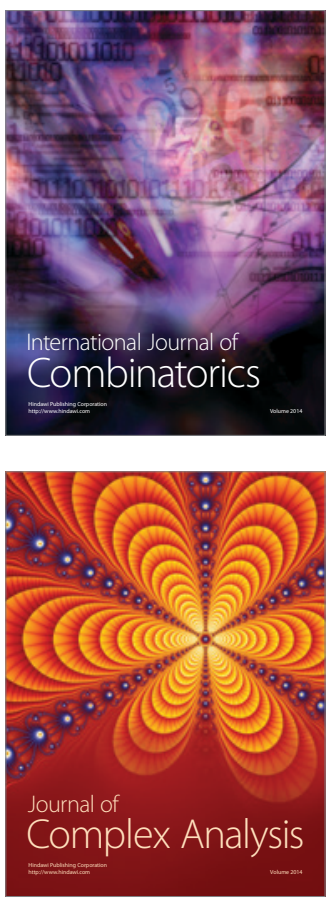

International Journal of

Mathematics and

Mathematical

Sciences
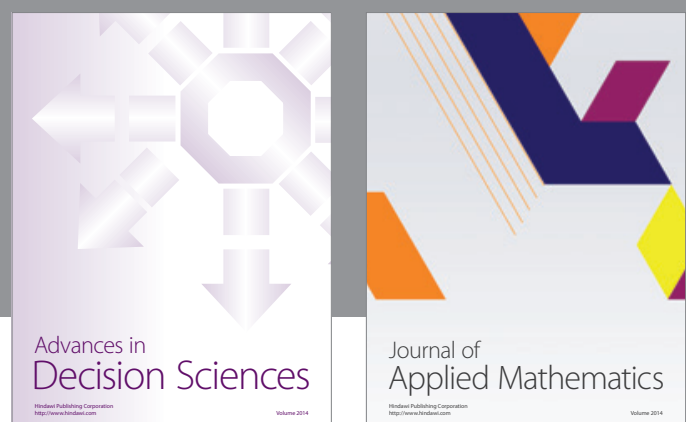

Journal of

Applied Mathematics
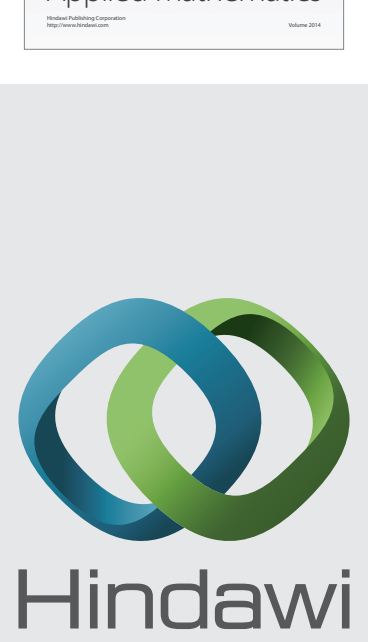

Submit your manuscripts at http://www.hindawi.com
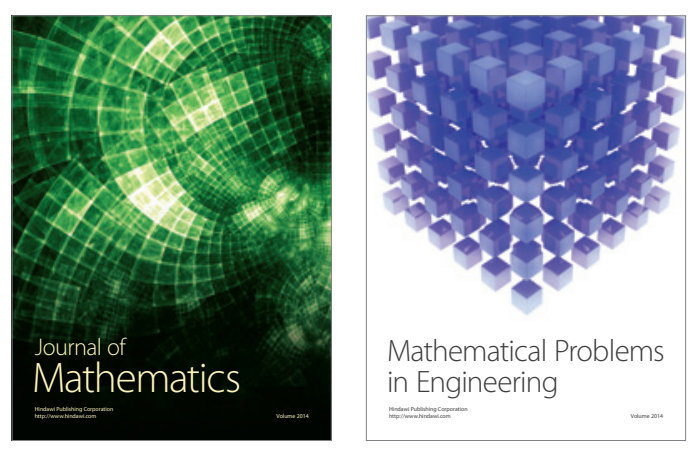

Mathematical Problems in Engineering
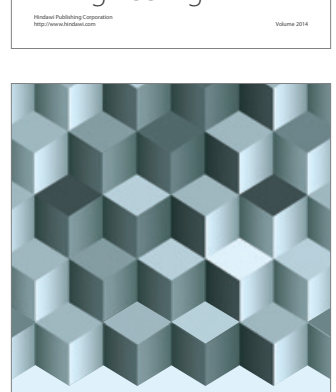

Journal of

Function Spaces
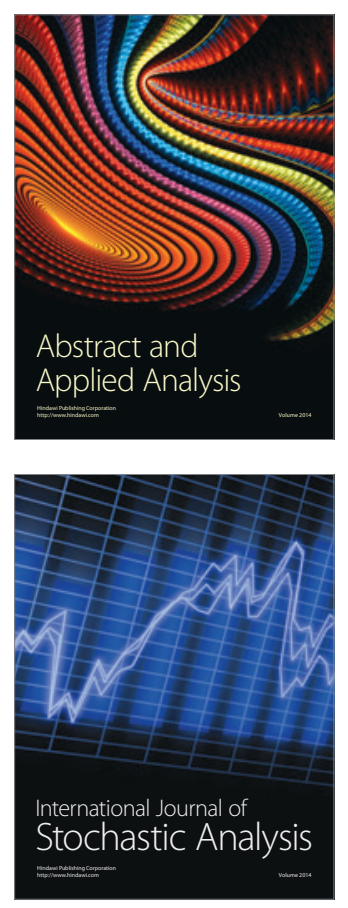

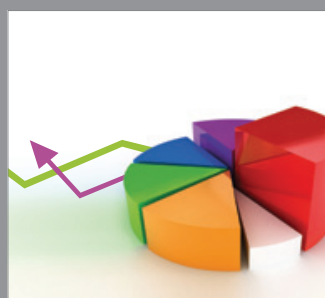

ournal of

Probability and Statistics

Promensencen
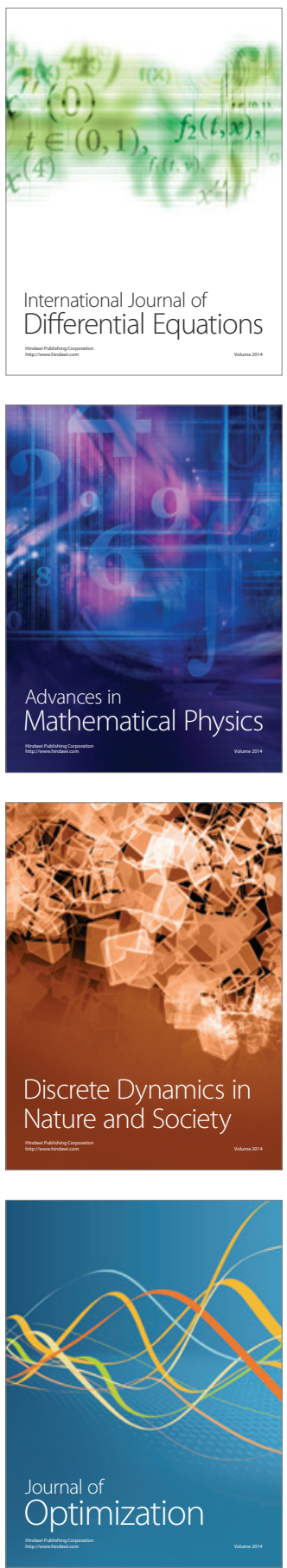